\title{
Approximate solutions of the Schrödinger equation with energy- dependent screened Coulomb potential in D - dimensions
}

\author{
Uduakobong Sunday Okorie ${ }^{1,3+}$, Akpan Ndem Ikot $^{2,3}{ }^{\oplus}$, Precious Ogbonda Amadi ${ }^{3}$, Alalibo Thompson \\ Ngiangia $^{3}{ }^{(-)}$, Etebong Emmanuel Ibekwe ${ }^{1,3([)}$
}

1. Department of Physics, Akwa Ibom State University, Ikot Akpaden P. M. B. 1167, Uyo, Nigeria

2. Department of Physics, University of South Africa, Florida 1710, Johannesburg, South Africa

3. Theoretical Physics Group, Department of Physics, University of Port Harcourt, P. M. B. 5323 Choba, Nigeria

${ }^{+}$Corresponding author: Uduakobong Sunday Okorie, Phone: +2347081545195 Email address: uduakobongokorie@aksu.edu.ng

\section{ARTICLE INFO}

Article history:

Received: October 26, 2019

Accepted: April 07, 2020

Published: October 01, 2020
Keywords:

1. Schrödinger equation

2. energy-dependent screened Coulomb Potential

3. Nikiforov-Uvarov method

4. Greene-Aldrich approximation scheme
ABSTRACT: Within the framework of the conventional NikiforovUvarov method and a new form of Greene-Aldrich approximation scheme, we solved the Schrödinger equation with the energydependent screened Coulomb potential. Energy eigenvalues and energy eigenfunctions were obtained both approximately and numerically at different dimensions. The energy variations with different potential parameters, quantum numbers and energy slope parameter, respectively were also discussed graphically. The major finding of this research is the effect of the energy slope parameter on the energy spectra, which is seen in the existence of two simultaneous energy values for a particular quantum state. Our special cases also agree with the results obtained from literature, when the energy slope parameter is zero.

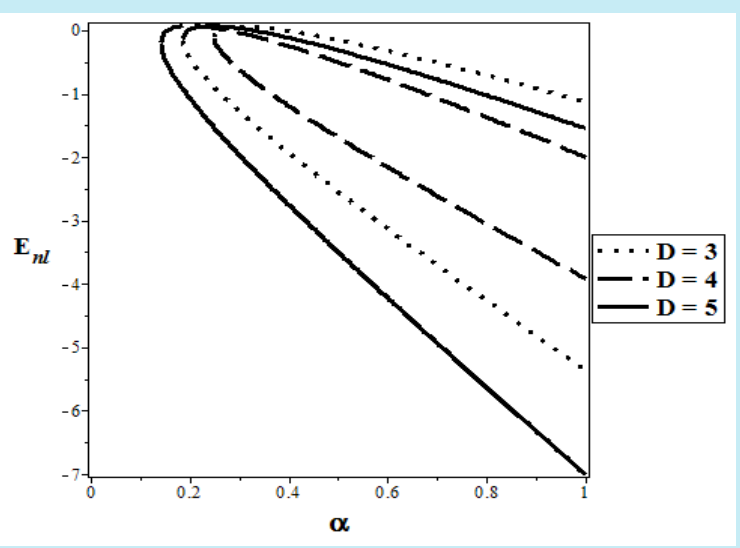

The Schrödinger equation with many physical potentials model have been investigated in recent times with different analytical methods such as Nikiforov-Uvarov (NU) method $^{5-8}$, asymptotic iteration method $(\mathrm{AIM})^{9-14}$, supersymmetric quantum mechanics(SUSYQM) ${ }^{15-18}$ among others $^{19-22}$. One of such potential models is the screened Coulomb potential, which is given by Eq. $1^{23}$.

$V(r)=-\frac{A e^{-\alpha r}}{r}$

The screened Coulomb potential, also known as the Yukawa potential is greatly important, with applications cutting across nuclear Physics and Condensed-matter Physics ${ }^{23}$. Here, its usage is involved in short-ranged interactions ${ }^{24-26}$. The screened-Coulomb potential is known to be the is because it contains all the necessary information needed for the full description of a quantum state such as the probability density and entropy of the system ${ }^{4}$. 
potential of a charged particle in a weakly nonideal plasma. It also describes the charged particle effects in a sea of conduction electrons in solidstate physics ${ }^{27}$.

An approximate solution of the Schrödinger equation interacting with an inversely quadratic Yukawa potential has been obtained using SUSYQM $^{15}$, where the screened Coulomb potential was obtained as a special case by varying the potential strength. Also, an approximate analytical solution of the radial Schrödinger equation for the screened Coulomb potential has been obtained, with energy eigenvalues and its corresponding eigenfunctions computed in closed forms ${ }^{28}$.

Several researchers have also devoted great attention to investigate the quantum systems of the energy dependence of different potentials ${ }^{29-31}$. Hassanabadi et al ${ }^{32,33}$ studied the exact solutions of D-dimensional Schrödinger and Klein-Gordon equations using the Nikiforov-Uvarov method. Also, Lombard et al..$^{34}$ investigated the wave equation energy-dependent potential for confined systems. Numerous applications of the energydependent potential of wave equations have been seen in the spectrum of confined systems and heavy quark confinement in nuclear and molecular physics ${ }^{35,36}$. Recently, Budaca ${ }^{37}$ studied an energy-dependent Coulomb-like potential within the framework of Bohr Hamiltonian. The author further reported that the energy dependence on the coupling constant of the potential drastically changes the analytical properties of wave function and the corresponding eigenvalues of the system. Also, Boumali and Labidi ${ }^{38}$ investigated the Shannon and Fisher information in the Klein-Gordon equation with energydependent potential.

In this research, we seek to investigate the influence of the energy-dependent screened

Coulomb potential defined as in Eq. 2,

$V\left(r, E_{n \ell}\right)=-\frac{A\left(1+g E_{n \ell}\right) e^{-\alpha r}}{r}$

where $g$ is the energy slope parameter, $A$ is the depth of the potential, and $\alpha$ is the range of the potential. The effects of the energy dependence on the screened Coulomb potential have not been considered before in any literature, to the best of our knowledge. It can be deduced that when $g=$ 0 , the potential of Eq. 2 reduces to the Screened Coulomb potential. When $g=0$ as $\alpha \rightarrow 0$, the potential of Eq. 2 reduces to the Coulomb potential. Using the conventional NU method, we will derive the $\ell$-wave bound state solutions and their eigenfunctions of the Schrödinger equation for the energy-dependent screened Coulomb potential, both analytically and numerically. Special cases are also considered and our results are compared with existing literature for confirmation sake.

The organization of this work is as follows: In section 2, we determine the eigensolutions of the energy-dependent screened Coulomb potential by employing a new form of Greene-Aldrich approximation scheme and Nikiforov-Uvarov method. Section 3 is devoted to discuss the results obtained and compare to results in relevant literature. The conclusion of the work is presented in section 4 .

\section{Bound state solution of the energy-dependent screened Coulomb potential}

The radial part of the Schrödinger equation in D-dimension ${ }^{39}$ is given by Eq. 3.

$\psi^{\prime \prime}(r)+\frac{2 \mu}{\hbar^{2}}\left(E_{n \ell}-V(r)\right) \psi(r)+\frac{1}{r^{2}}\left[\frac{(D-1)(D-3)}{4}+\ell(\ell+D-2)\right] \psi(r)=0$

where $\mu$ is the reduced mass, $E_{n \ell}$ is the non-relativistic energy eigenvalues to be determined. Substituting Eq. 2 into Eq. 3 gives Eq. 4.

$\psi^{\prime \prime}(r)+\frac{2 \mu}{\hbar^{2}}\left(E_{n \ell}+\frac{A\left(1+g E_{n \ell}\right) e^{-\alpha r}}{r}\right) \psi(r)+\frac{1}{r^{2}}\left[\frac{(D-1)(D-3)}{4}+\ell(\ell+D-2)\right] \psi(r)=0$

In solving Eq. 4, we invoke a new form of Greene-Aldrich approximation scheme ${ }^{23}$ to deal with the centrifugal term since $\ell \neq 0$. The approximations schemes are given by Eq. 5 and 6 . 
$f_{1}(r)=\frac{1}{r^{2}} \approx \frac{4 \alpha^{2} e^{-2 \alpha r}}{\left(1-e^{-2 \alpha r}\right)^{2}}=f_{2}(r)$

$\frac{1}{r^{2}} \approx \frac{4 \alpha^{2} e^{-2 \alpha r}}{\left(1-e^{-2 \alpha r}\right)^{2}}+\frac{\alpha^{2}}{3}=f_{3}(r)$

The plots explaining the rationality and validity of the above Eq. 5 and 6 and their expansions are given in Fig. 1(a-d). Figure 1a shows the plots of $f_{1}(r), f_{2}(r)$ and $f_{3}(r)$ as they vary with $r$, when the screening parameter $\alpha$ is taken to be 0.5 . In addition, we employ $\frac{1}{r}$ from $f_{1}(r)$ as given in Eq. 7 .

$f_{4}(r)=\frac{1}{r} \approx \frac{2 \alpha e^{-\alpha r}}{1-e^{-2 \alpha r}} \equiv f_{5}(r)$

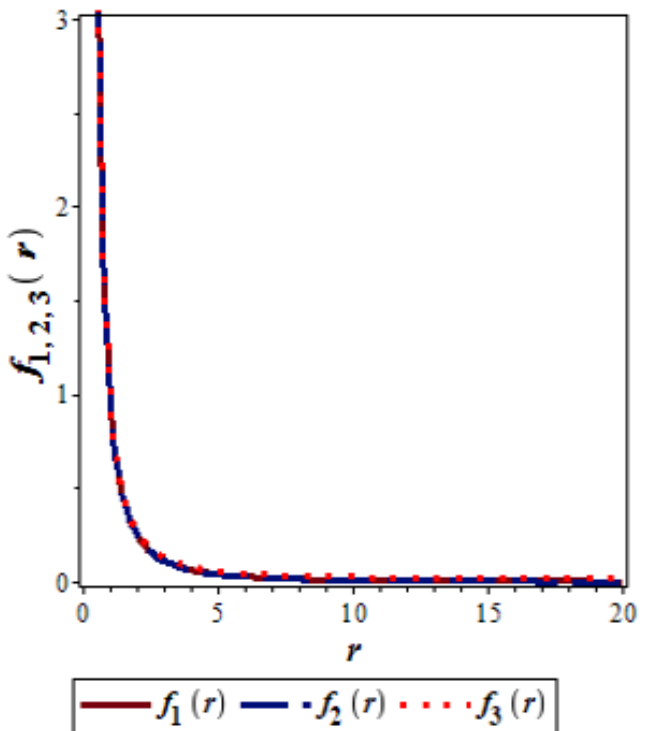

(a)

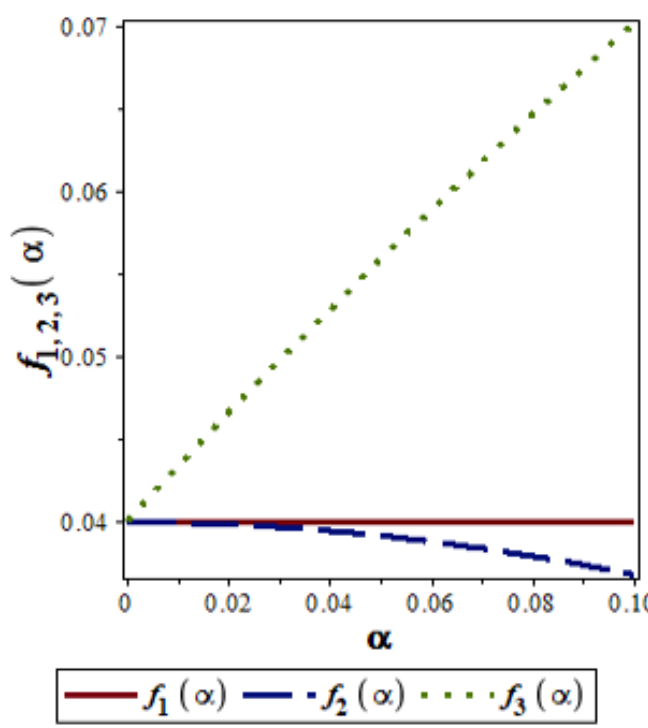

(c)

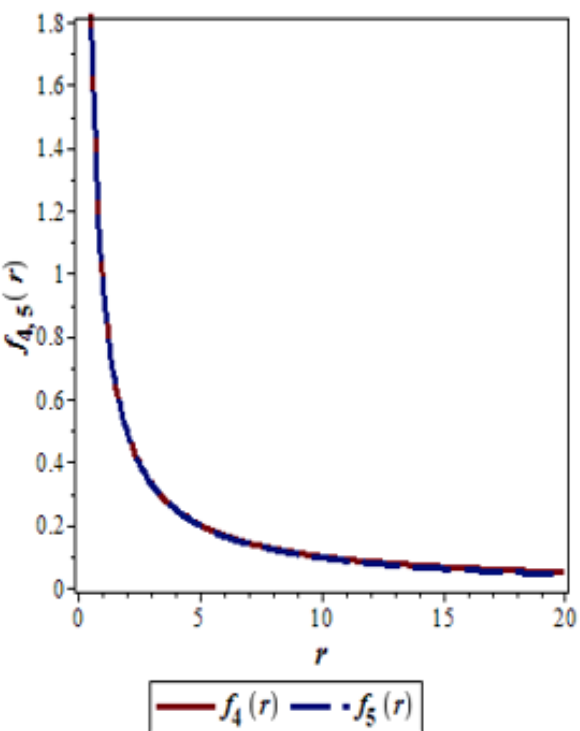

(b)

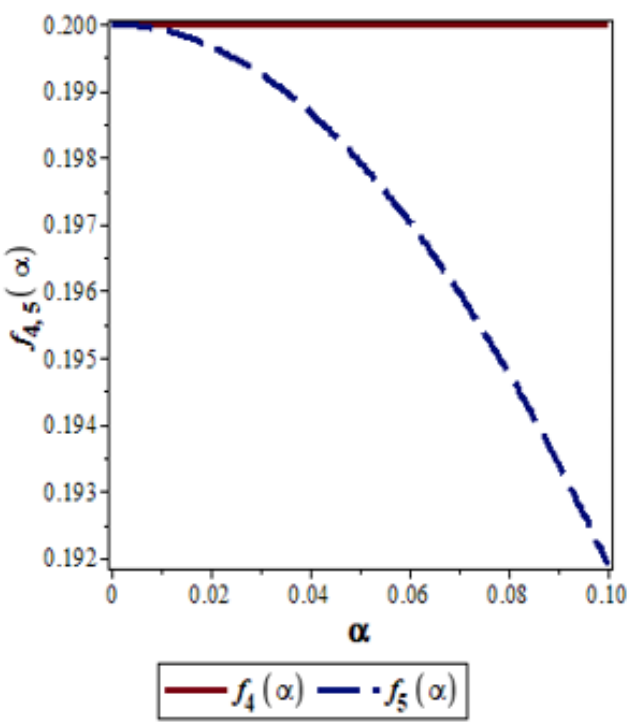

(d)

Figure 1. The plots of expressions (a) $f_{1}(r), f_{2}(r), f_{3}(r)$ as functions of $r$ with $\alpha=0.5$; (b) $f_{4}(r)$, $f_{5}(r)$ as functions of $r$ with $\alpha=0.5$; (c) $f_{1}(\alpha), f_{2}(\alpha), f_{3}(\alpha)$ as functions of $\alpha$ with $r=5$; (d) $f_{4}(\alpha), f_{5}(\alpha)$ as functions of $\alpha$ with $r=5 n m$. 
Here, we have ignored the second expansion term $\frac{\alpha\left(1-e^{-2 \alpha r}\right)}{\left(12 e^{-\alpha r}\right)}$ when expanding Eq. 6. Furthermore, the rationality and validity of this expansion is given in Fig. $1 \mathrm{~b}$, with $\alpha=0.5$. Considering the plots of the above expressions as function ns of the screening parameter $\alpha$ as shown in Figs. 1c and 1d, we observe that the approximation $f_{3}(\alpha)$ corresponds to that of $f_{1}(\alpha)$ when $\alpha \leq 0.05$. As such, we see that the approximation $f_{3}(\alpha)$ is better than that of $f_{2}(\alpha)$.

With the above approximation schemes, Eq. 4 becomes Eq. 8.

$\psi^{\prime \prime}(r)+\left[\frac{2 \mu E_{n \ell}}{\hbar^{2}}+\frac{4 \mu A\left(1+g E_{n \ell}\right) e^{-2 \alpha r}}{\hbar^{2}\left(1-e^{-2 \alpha r}\right)}-\frac{4 \alpha^{2} \gamma e^{-2 \alpha r}}{\hbar^{2}\left(1-e^{-2 \alpha r}\right)^{2}}-\frac{\gamma \alpha^{2}}{3}\right] \psi(r)=0$

where $\gamma$ is given by Eq. 9 .

$\gamma=\left[\frac{(D-1)(D-3)}{4}+\ell(\ell+D-2)\right]$

By using the coordinate transformation of Eq. 10.

$z=e^{-2 \alpha r}$

Eq. 8 becomes the differential equation of the form given in Eq. 11.

$\psi^{\prime \prime}(z)+\frac{(1-z)}{z(1-z)} \psi^{\prime}(z)+\frac{1}{z^{2}(1-z)^{2}}\left[-\left(\varepsilon^{2}+\beta^{2}\right) z^{2}+\left(2 \varepsilon^{2}+\beta^{2}-\gamma\right) z-\varepsilon^{2}\right] \psi(z)=0$

where $\varepsilon^{2}$ is given by Eq. 12 .

$\varepsilon^{2}=-\left(\frac{\mu E_{n \ell}}{2 \hbar^{2} \alpha^{2}}-\frac{\gamma}{12}\right) ; \beta^{2}=\frac{\mu A\left(1+g E_{n \ell}\right)}{\hbar^{2} \alpha}$

By comparing Eq. 11 and Eq. A1 (see Appendix), we have the following parameters, Eq. 13:

$\begin{aligned} \tilde{\tau}(z) & =1-z \\ \sigma(z) & =z(1-z) \\ \tilde{\sigma}(z)=-\left(\varepsilon^{2}+\beta^{2}\right) z^{2}+\left(2 \varepsilon^{2}+\beta^{2}-\gamma\right) z-\varepsilon^{2} & \end{aligned}$

Substituting Eq. 13 into Eq. A8 (see Appendix), we get $\pi(z)$, Eq. 14:

$\pi(z)=-\frac{z}{2} \pm \sqrt{(a-k) z^{2}+(k+b) z+c}$

where $a, b$ and $c$ are given by Eq. 15 .

$a=\frac{1}{4}+\varepsilon^{2}+\beta^{2}$

$b=\gamma-2 \varepsilon^{2}-\beta^{2}$

$c=\varepsilon^{2}$

We can obtain the constant $k$, by expressing the discriminant under the square root of Eq. 14 to be equal to zero. As such, we have Eq. 16 
$k_{ \pm}=-\left(\gamma-\beta^{2}\right) \pm 2 \sqrt{\varepsilon^{2}} \sqrt{\left(\frac{1}{4}+\gamma\right)}$

Substituting Eq. 16 into Eq. 14 yields Eq. 17:

$\pi(z)=-\frac{z}{2} \pm\left\{\begin{array}{l}\left(\sqrt{\varepsilon^{2}}-\sqrt{\frac{1}{4}+\gamma}\right) z-\sqrt{\varepsilon^{2}} ; \text { fork }_{+}=-\left(\gamma-\beta^{2}\right)+2 \sqrt{\varepsilon^{2}} \sqrt{\left(\frac{1}{4}+\gamma\right)} \\ \left(\sqrt{\varepsilon^{2}}-\sqrt{\frac{1}{4}+\gamma}\right) z+\sqrt{\varepsilon^{2}} ; \text { fork }_{-}=-\left(\gamma-\beta^{2}\right)-2 \sqrt{\varepsilon^{2}} \sqrt{\left(\frac{1}{4}+\gamma\right)}\end{array}\right.$

According to NU method ${ }^{40}$, we choose the expression $\pi(z)_{-}$which the function $\tau(z)$ has a negative derivative. This is given by Eq. 18

$\pi(z)_{-}=-\left(\frac{1}{2}+\sqrt{\varepsilon^{2}}+\sqrt{\frac{1}{4}+\gamma}\right) z-\sqrt{\varepsilon^{2}}$

with $\tau(z)$ being obtained with Eq. 19.

$\tau(z)=1-2 \sqrt{\varepsilon^{2}}-2\left(1+2 \sqrt{\varepsilon^{2}}+\sqrt{\frac{1}{4}+\gamma}\right) z$

By recalling Eq. A9 (see Appendix), we define the constant $\lambda$ (Eq. 20) as

$\lambda=-\left(\gamma-\beta^{2}\right)-2 \sqrt{\varepsilon^{2}} \sqrt{\frac{1}{4}+\gamma}-\left(\frac{1}{2}+\sqrt{\varepsilon^{2}}+\sqrt{\frac{1}{4}+\gamma}\right)$

Substituting Eq. 20 into Eq. A10 (see Appendix) and carrying out algebraic simplifications, where $\tau^{\prime}(\mathrm{z})$ is given by Eq. 21

$\tau^{\prime}(z)=-2\left(1+\sqrt{\varepsilon^{2}}+\sqrt{\frac{1}{4}+\gamma}\right)$

and $\sigma^{\prime \prime}(\mathrm{z})$, Eq. 22

$\sigma^{\prime \prime}(z)=-2$

we obtain Eq. 23

$\varepsilon^{2}=\frac{1}{4}\left[\frac{(n+\chi)^{2}-\beta^{2}}{(n+\chi)}\right]^{2}$

where $\chi$ is given by Eq. 24

$\chi=\frac{1}{2}(1+\sqrt{1+4 \gamma})$

Substituting Eq. 12 into Eq. 23 yields a complicated transcendental energy eigenvalue equation of the energy dependent screened Coulomb potential in D-dimensions as (Eq. 25):

$\left[(n+\chi)-\frac{\mu A\left(1+g E_{n \ell}\right)}{\hbar^{2} \alpha(n+\chi)}\right]^{2}+\frac{2 \mu E_{n \ell}}{\hbar^{2} \alpha^{2}}-\frac{\gamma}{3}=0$ 
Eq. 25 can also be expressed by Eq. 26.

$E_{n \ell}=\frac{-\hbar^{2} \alpha^{2}}{2 \mu}\left[\left((n+\chi)-\frac{\mu A\left(1+g E_{n \ell}\right)}{\hbar^{2} \alpha(n+\chi)}\right)^{2}-\frac{\gamma}{3}\right]$

where $\gamma$ and $\chi$ are given in Eqs. 9 and 24, respectively.

To obtain the special case, we first rewrite Eq. 26 to the form of Eq. 27:

$E_{n \ell}=\frac{-\hbar^{2}}{2 \mu}\left[\left(\alpha(n+\chi)-\frac{\mu A\left(1+g E_{n \ell}\right)}{\hbar^{2}(n+\chi)}\right)^{2}-\frac{\alpha^{2} \gamma}{3}\right]$

As $\alpha \rightarrow 0$ and $g \rightarrow 0$, Eq. 2 reduces to the standard Coulomb potential of the form of Eq. 28.

$V(r)=-\frac{A}{r}$

Setting the parameters $D=3$ as $\alpha \rightarrow 0$ and $\rightarrow 0$, we obtain the energy eigenvalue equation (Eq. 29):

$E_{n \ell}=-\frac{\mu A^{2}}{2 \hbar^{2}(n+\ell+1)^{2}}$

This result is very consistent with the result obtained in Eq. 101 of Birkdemir et al.${ }^{41}$. Also, taking the natural units $\left(\hbar^{2}=\mu=1\right)$ and setting $D=3 a n d g=0$, the energy eigenvalue expression of Eq. 26 can be reduced to Eq. 30 .

$E_{n \ell}=\frac{-\alpha^{2}}{2}\left[\left((n+\ell+1)-\frac{A}{\alpha(n+\ell+1)}\right)^{2}-\frac{\ell(\ell+1)}{3}\right]$

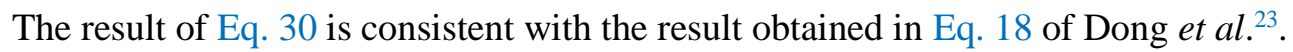

To obtain the corresponding wave functions, we substitute $\pi(z) \_$and $\sigma(z)$ from Eqs. 18 and 13, respectively into Eq. A4 (see Appendix) and solve the first-order differential equation. This gives Eq. 31 .

$\Phi(z)=z^{\sqrt{\varepsilon^{2}}}(1-z)^{\frac{1}{2}+\sqrt{\frac{1}{4}+\gamma}}$

The weight function $\rho(z)$ from Eq. A6 (see Appendix) can be obtained Eq. 32

$\rho(z)=z^{2^{\sqrt{\varepsilon^{2}}}}(1-z)^{2 \sqrt{\frac{1}{4}+\gamma}}$

From the Rodrigues relation of Eq. A5 (see Appendix), we obtain Eq. 33 and Eq. 34.

$y_{n}(z)=B_{n} z^{-2 \sqrt{\varepsilon^{2}}}(1-z)^{-2 \sqrt{\frac{1}{4}+\gamma}} \frac{d^{n}}{d z^{n}}\left[z^{n+2 \sqrt{\varepsilon^{2}}}(1-z)^{n+2 \sqrt{\frac{1}{4}+\gamma}}\right]$

$y_{n}(z) \equiv B_{n} P_{n}^{\left(2 \sqrt{\varepsilon^{2}}, 2 \sqrt{\frac{1}{4}+\gamma}\right)}(1-2 z)$

where $P_{n}^{(\theta, \vartheta)}$ is the Jacobi Polynomial.

Substituting $\Phi(z)$ and $y_{n}(z)$ from Eqs. 31 and 34, respectively into Eq. A2 (see Appendix), we obtain Eq. 35: 
$\psi(z)=B_{n} z^{\sqrt{\varepsilon^{2}}}(1-z)^{G} P_{n}^{\left(2 \sqrt{\varepsilon^{2}}, 2 G-1\right)}(1-2 z)$

where $G$ is given by Eq. 36 .

$G=\frac{1}{2}+\sqrt{\frac{1}{4}+\gamma}$

From the definition of the Jacobi Polynomials ${ }^{42}$ results Eq. 37.

$P_{n}^{(\theta, \vartheta)}(\omega)=\frac{\Gamma(n+\theta+1)}{n ! \Gamma(\theta+1)}{ }_{2} F_{1}\left(-n, \theta+\vartheta+n+1, \theta+1 ; \frac{1-\omega}{2}\right)$

In terms of hypergeometric Polynomials, Eq. 35 can be written as Eq. 38.

$\psi(z)=B_{n} z^{\sqrt{\varepsilon^{2}}}(1-z)^{G} \frac{\Gamma\left(n+2 \sqrt{\varepsilon^{2}}+1\right)}{n ! \Gamma\left(2 \sqrt{\varepsilon^{2}}+1\right)}{ }_{2} F_{1}\left(-n, 2 \sqrt{\varepsilon^{2}}+2 G+n, 2 \sqrt{\varepsilon^{2}}+1 ; z\right)$.

\section{Results and Discussion}

In this study, the energy eigenvalues for the energy-dependent screened Coulomb potential as they vary with the screening parameter were computed for different quantum states as shown in Tab. 1. The existence of the energy slope parameter in Eq. 26 results in two different energy spectra for a particular quantum state and a specific screening parameter. The screening parameter varies in an inverse version with the duo energy spectra. The energy eigenvalues for the energy-dependent screened Coulomb potential as a function of screening parameters in higher dimensions were also computed, as shown in Tabs. 2 and 3, respectively. By employing Eq. 30, we have also computed the energy eigenvalues in three dimensions and in the absence of the energy slope parameter, as shown in Tab. 4. For the two different potential depths considered, we obtained the bound state energy eigenvalues for different quantum states of $\mathbf{2 p - 4 f}$, as they vary with different screening parameters. It can be seen that the energy eigenvalues for the different potential depths decrease as the screening parameter increases, at each quantum state. Our analytical result of Eq. 30 and its corresponding numerical results of Tab. 4 are very consistent with the results obtained by Dong et al. ${ }^{23}$. We have also computed the energy eigenvalues of Eq. 26 for higher dimensions, as shown in Tabs. 5 and 6, respectively. The level of effects imposed by the potential parameters of Eq. 2 on the energy eigenvalues of Eq. 26 are shown in Figs. 2-7. For different dimensions, the energy slope parameter causes an interwoven interaction of the curves, as compared to the situation when the energy slope parameter is zero. Figs. 8 and 9 show the variation of energy eigenvalues for the energy-dependent screened Coulomb potential with the different quantum numbers, for different values energy slope parameter considered. The trend of the relationship shows that the energy eigenvalues decrease as the quantum numbers increase. In Fig. 10 , we also plotted the variation of the energy eigenvalues with the energy slope parameter for different dimensions.

Table 1. Eigenvalues $\left(E_{n \ell}\right)$ for the energy-dependent screened Coulomb potential as a function of the parameter $\alpha$ for $2 p-4 f$ states, with $D=3$.

\begin{tabular}{|c|c|c|c|}
\hline States & $\alpha$ & $\boldsymbol{E}_{\boldsymbol{n} \boldsymbol{\ell}}(\boldsymbol{g}=-\mathbf{1}, \boldsymbol{A}=\mathbf{2})$ & $\boldsymbol{E}_{\boldsymbol{n} \boldsymbol{\ell}}(\boldsymbol{g}=\mathbf{1}, \boldsymbol{A}=\mathbf{2})$ \\
\hline \multirow{3}{*}{$2 p$} & 0.025 & $-0.05000000000,-0.9484636700$ & $-0.2469390303,-3.653060970$ \\
\cline { 2 - 4 } & 0.050 & $-0.1000000000,-0.8934950102$ & $-0.2261820091,-3.573817991$ \\
\hline & 0.075 & $-0.1499999989,-0.8344159637$ & $-0.2056916349,-3.494308363$ \\
\hline
\end{tabular}




\begin{tabular}{|c|c|c|c|}
\hline \multirow{3}{*}{$3 p$} & 0.025 & $-0.3282430205,-2.396756978$ & $-0.1279838429,-6.147016156$ \\
\cline { 2 - 4 } & 0.050 & $-0.2185168124,-2.731483190$ & $-0.1003205303,-5.949679472$ \\
\hline \multirow{3}{*}{$3 d$} & 0.075 & $-0.1419248550,-3.033075144$ & $-0.07486233817,-5.750137661$ \\
\hline \multirow{3}{*}{$4 p$} & 0.025 & $-0.3273369696,-2.397663029$ & $-0.1276723472,-6.147327652$ \\
\cline { 2 - 4 } & 0.050 & $-0.2155358280,-2.734464174$ & $-0.09903861948,-5.950961383$ \\
\hline \multirow{3}{*}{$4 d$} & 0.075 & $-0.1360998141,-3.038900185$ & $-0.07189047036,-5.753109529$ \\
\hline \multirow{3}{*}{4} & 0.025 & $-0.1013443775,-6.298655622$ & $-0.06696010299,-9.533039897$ \\
\hline \multirow{3}{*}{$4 f$} & 0.050 & $-0.05236401820,-6.747635982$ & $-0.03856747648,-9.161432524$ \\
\hline & 0.075 & $-0.02019553609,-7.179804460$ & $-0.01650824116,-8.783491756$ \\
\hline & 0.025 & $-0.1008065565,-6.299193443$ & $-0.06660798158,-9.533392018$ \\
\hline & 0.050 & $-0.05037315511,-6.749626845$ & $-0.03710618138,-9.162893819$ \\
\hline & 0.075 & $-0.01600781253,-7.183992184$ & $-0.01308764621,-8.786912351$ \\
\hline & 0.025 & $-0.1000000000,-6.300000000$ & $-0.06607984858,-9.533920151$ \\
\hline & 0.050 & $-0.04738907715,-6.752610923$ & $-0.03491511579,-9.165084884$ \\
\hline
\end{tabular}

Table 2. Eigenvalues $\left(E_{n \ell}\right)$ for the energy-dependent screened Coulomb potential as a function of the parameter $\alpha$ for $\mathbf{2 p}-\mathbf{4} \boldsymbol{f}$ states, with $D=4$.

\begin{tabular}{|c|c|c|c|}
\hline States & $\alpha$ & $\boldsymbol{E}_{\boldsymbol{n} \boldsymbol{\ell}}(\boldsymbol{g}=-\mathbf{1}, \boldsymbol{A}=\mathbf{2})$ & $\boldsymbol{E}_{\boldsymbol{n} \boldsymbol{\ell}}(\boldsymbol{g}=\mathbf{1}, \boldsymbol{A}=\mathbf{2})$ \\
\hline \multirow{3}{*}{$2 p$} & 0.025 & $-0.6406250000,-0.6619912551$ & $-0.1771068474,-4.791643153$ \\
\cline { 2 - 4 } & 0.050 & $-0.7187500000,-0.4363825014$ & $-0.1516973143,-4.660802686$ \\
\cline { 2 - 4 } & 0.075 & $-0.5523050676,-1.041444930$ & $-0.1269955847,-4.529254413$ \\
\hline \multirow{3}{*}{$3 p$} & 0.025 & $-0.1676529658,-4.263597033$ & $-0.09251657939,-7.726233419$ \\
\cline { 2 - 4 } & 0.050 & $-0.1017568786,-4.635743120$ & $-0.06332496524,-7.449175033$ \\
\hline \multirow{3}{*}{$3 d$} & 0.075 & $-0.05426252476,-4.989487474$ & $-0.03776841503,-7.168481584$ \\
\hline \multirow{3}{*}{$4 p$} & 0.025 & $-0.1668742692,-4.264375729$ & $-0.09209870569,-7.726651293$ \\
\hline \multirow{3}{*}{$4 d$} & 0.050 & $-0.09894423101,-4.638555767$ & $-0.06159768496,-7.450902314$ \\
\hline \multirow{3}{*}{$4 d$} & 0.075 & $-0.04845181230,-4.995298186$ & $-0.03374430925,-7.172505689$ \\
\hline \multirow{3}{*}{$4 f$} & 0.025 & $-0.06465429287,-8.566595705$ & $-0.04786732380,-11.57088267$ \\
\hline & 0.050 & $-0.02501719398,-9.112482806$ & $-0.02055263540,-11.09194736$ \\
\hline & 0.075 & $-0.002313419520,-9.641436577$ & $-0.002103392985,-10.60414660$ \\
\hline & 0.025 & $-0.06403407537,-8.567215922$ & $-0.04740969809,-11.57134030$ \\
\hline & 0.050 & $-0.02269659485,-9.114803405$ & $-0.01864771539,-11.09385228$ \\
\hline & 0.075 & $0.002607849543,-9.646357846$ & $0.002371302471,-10.60862130$ \\
\hline
\end{tabular}

Table 3. Eigenvalues $\left(E_{n \ell}\right)$ for the energy-dependent screened Coulomb potential as a function of the parameter $\alpha$ for $\mathbf{2 p}-\mathbf{4} \boldsymbol{f}$ states, with $D=5$.

\begin{tabular}{|c|c|c|c|}
\hline States & $\alpha$ & $E_{n \ell}(g=-1, A=2)$ & $E_{n \ell}(g=1, A=2)$ \\
\hline \multirow{3}{*}{$2 p$} & 0.025 & $-0.3273369696,-2.397663029$ & $-0.1276723472,-6.147327652$ \\
\hline & 0.050 & $-0.2155358280,-2.734464174$ & $-0.09903861948,-5.950961383$ \\
\hline & 0.075 & $-0.1360998141,-3.038900185$ & $-0.07189047036,-5.753109529$ \\
\hline \multirow{3}{*}{$3 p$} & 0.025 & $-0.1008065565,-6.299193443$ & $-0.06660798158,-9.533392018$ \\
\hline & 0.050 & $-0.05037315511,-6.749626845$ & $-0.03710618138,-9.162893819$ \\
\hline & 0.075 & $-0.01600781253,-7.183992184$ & $-0.01308764621,-8.786912351$ \\
\hline \multirow{3}{*}{$3 d$} & 0.025 & $-0.1000000000,-6.300000000$ & $-0.06607984858,-9.533920151$ \\
\hline & 0.050 & $-0.04738907715,-6.752610923$ & $-0.03491511579,-9.165084884$ \\
\hline & 0.075 & $-0.009735385820,-7.190264611$ & $-0.007961748818,-8.792038248$ \\
\hline \multirow{3}{*}{$4 p$} & 0.025 & $-0.4194183076,-11.08305817$ & $-0.03358353903,-13.84141646$ \\
\hline & 0.050 & $-0.009315896675,-11.74068410$ & $-0.008259866067,-13.24174013$ \\
\hline & 0.075 & $0.005363836706,-12.38036383$ & $0.005257711402,-12.63025771$ \\
\hline \multirow{3}{*}{$4 d$} & 0.025 & $-0.04123429366,-11.08376571$ & $-0.03301776018,-13.84198224$ \\
\hline & 0.050 & $-0.006652702848,-11.74334730$ & $-0.005898852562,-13.24410115$ \\
\hline & 0.075 & $0.01103813411,-12.38603813$ & $0.01081993497,-12.63581993$ \\
\hline \multirow{3}{*}{$4 f$} & 0.025 & $-0.04029105188,-11.08470895$ & $-0.03226346048,-13.84273654$ \\
\hline & 0.050 & $-0.003103656681,-11.74689634$ & $-0.002752143970,-13.24724786$ \\
\hline & 0.075 & $0.01859579365,-12.39359579$ & $0.01822863105,-12.64322863$ \\
\hline
\end{tabular}


Table 4. Eigenvalues $\left(E_{n \ell}\right)$ of Eq. 29 as a function of the parameter $\alpha$ for $\mathbf{2 p}-\mathbf{4} \boldsymbol{f}$ states, with $D=3 a n d g=0$.

\begin{tabular}{|c|c|c|c|}
\hline States & $\alpha$ & $E_{n \ell}(A=2.0)$ & $E_{n \ell}(A=5.0)$ \\
\hline \multirow{3}{*}{$2 p$} & 0.025 & -0.4510416666 & -3.001041666 \\
\hline & 0.050 & -0.4041666666 & -2.879166666 \\
\hline & 0.075 & -0.3593750004 & -2.759375001 \\
\hline \multirow{3}{*}{$3 p$} & 0.025 & -0.1748263890 & -1.266493056 \\
\hline & 0.050 & -0.1326388888 & -1.149305555 \\
\hline & 0.075 & -0.09565972225 & -1.037326388 \\
\hline \multirow{3}{*}{$3 d$} & 0.025 & -0.1744097223 & -1.266076389 \\
\hline & 0.050 & -0.1309722221 & -1.147638888 \\
\hline & 0.075 & -0.09190972225 & -1.033576388 \\
\hline \multirow{3}{*}{$4 p$} & 0.025 & -0.07979166665 & -0.6610416665 \\
\hline & 0.050 & -0.04416666666 & -0.5504166665 \\
\hline & 0.075 & -0.01812500002 & -0.4493750000 \\
\hline \multirow{3}{*}{$4 d$} & 0.025 & -0.07937500000 & -0.6606250000 \\
\hline & 0.050 & -0.04250000000 & -0.5487500000 \\
\hline & 0.075 & -0.01437500002 & -0.4456250002 \\
\hline \multirow{3}{*}{$4 f$} & 0.025 & -0.07875000000 & -0.6600000000 \\
\hline & 0.050 & -0.04000000000 & -0.5462500000 \\
\hline & 0.075 & -0.008750000020 & -0.4400000002 \\
\hline
\end{tabular}

Table 5. Eigenvalues $\left(E_{n \ell}\right)$ of Eq. 25 as a function of the parameter $\alpha$ for $\mathbf{2 p}-\mathbf{4} \boldsymbol{f}$ states, with $D=4 a n d g=0$.

\begin{tabular}{|c|c|c|c|}
\hline States & $\boldsymbol{\alpha}$ & $\boldsymbol{E}_{\boldsymbol{n} \boldsymbol{\ell}}(\boldsymbol{A}=\mathbf{2 . 0})$ & $\boldsymbol{E}_{\boldsymbol{n} \boldsymbol{\ell}}(\boldsymbol{A}=\mathbf{5 . 0})$ \\
\hline \multirow{3}{*}{$2 p$} & 0.025 & -0.2715625000 & -1.876562500 \\
\cline { 2 - 4 } & 0.050 & -0.2262500000 & -1.756250000 \\
\hline \multirow{3}{*}{$3 p$} & 0.075 & -0.1840625002 & -1.639062500 \\
\hline \multirow{3}{*}{$3 d$} & 0.025 & -0.1167028062 & -0.8988456630 \\
\cline { 2 - 4 } & 0.050 & -0.07701530615 & -0.7841581630 \\
\hline \multirow{3}{*}{$4 p$} & 0.075 & -0.04420280616 & -0.6763456635 \\
\hline \multirow{3}{*}{$4 d$} & 0.025 & -0.1161819728 & -0.8983248295 \\
\hline & 0.050 & -0.07493197280 & -0.6716581635 \\
\hline \multirow{3}{*}{$4 f$} & 0.075 & -0.03951530614 & -0.4982214506 \\
\hline & 0.025 & -0.05470293210 & -0.3910339505 \\
\hline & 0.050 & -0.02251543210 & -0.2957214510 \\
\hline & 0.025 & -0.002202932106 & -0.4977006172 \\
\hline & 0.050 & -0.05418209880 & -0.3889506171 \\
\hline & 0.025 & -0.02043209876 & -0.2910339509 \\
\hline & 0.050 & 0.002484567894 & -0.4969714506 \\
\hline
\end{tabular}

Table 6. Eigenvalues $\left(E_{n \ell}\right)$ of Eq. 26 as a function of the parameter $\alpha$ for $\mathbf{2 p}$-4 $\boldsymbol{f}$ states, with $D=5$ and $g=0$.

\begin{tabular}{|c|c|c|c|}
\hline States & $\alpha$ & $E_{n \ell}(A=2.0)$ & $E_{n \ell}(A=5.0)$ \\
\hline \multirow{3}{*}{$2 p$} & 0.025 & -0.1744097223 & -1.266076389 \\
\hline & 0.050 & -0.1309722221 & -1.147638888 \\
\hline & 0.075 & -0.09190972225 & -1.033576388 \\
\hline \multirow{3}{*}{$3 p$} & 0.025 & -0.07937500000 & -0.6606250000 \\
\hline & 0.050 & -0.04250000000 & -0.5487500000 \\
\hline & 0.075 & -0.01437500002 & -0.4456250002 \\
\hline \multirow{3}{*}{$3 d$} & 0.025 & -0.07875000000 & -0.6600000000 \\
\hline & 0.050 & -0.04000000000 & -0.5462500000 \\
\hline & 0.075 & -0.008750000020 & -0.4400000002 \\
\hline \multirow{3}{*}{$4 p$} & 0.025 & -0.03718750000 & -0.3821875000 \\
\hline & 0.050 & -0.008750000000 & -0.2787500000 \\
\hline & 0.075 & 0.005312500000 & -0.1896874998 \\
\hline \multirow{3}{*}{$4 d$} & 0.025 & -0.03656250000 & -0.3815625000 \\
\hline & 0.050 & -0.006250000000 & -0.2762500000 \\
\hline & 0.075 & 0.01093750000 & -0.1840624998 \\
\hline
\end{tabular}




\begin{tabular}{|c|c|c|c|}
\hline \multirow{3}{*}{$4 f$} & 0.025 & -0.03572916666 & -0.3807291666 \\
\cline { 2 - 4 } & 0.050 & -0.002916666666 & -0.2729166666 \\
\hline
\end{tabular}

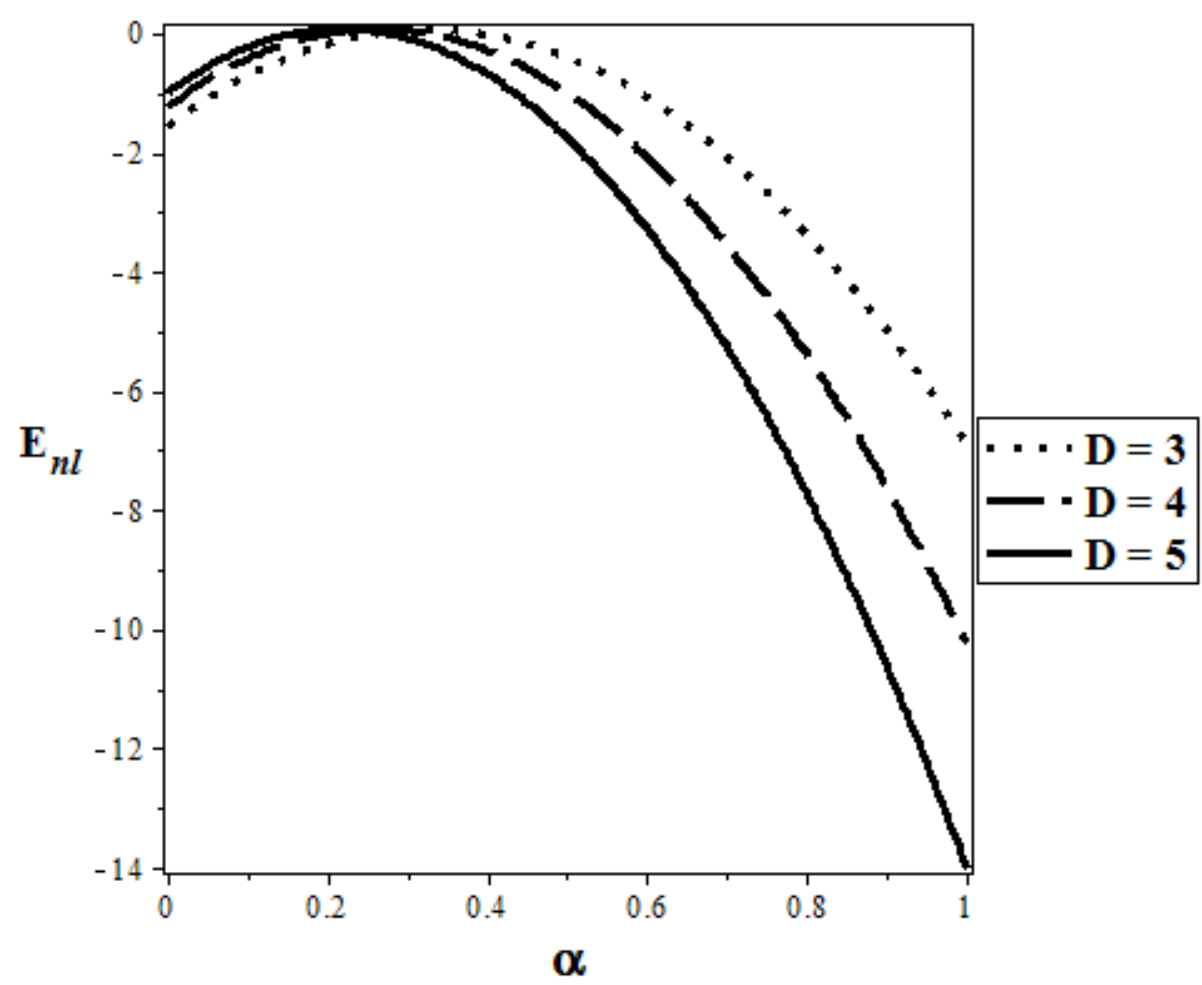

Figure 2. Energy Eigenvalue Variation with $\alpha$ for different dimensions with $g=0, A=5, n=2, \ell=1$.

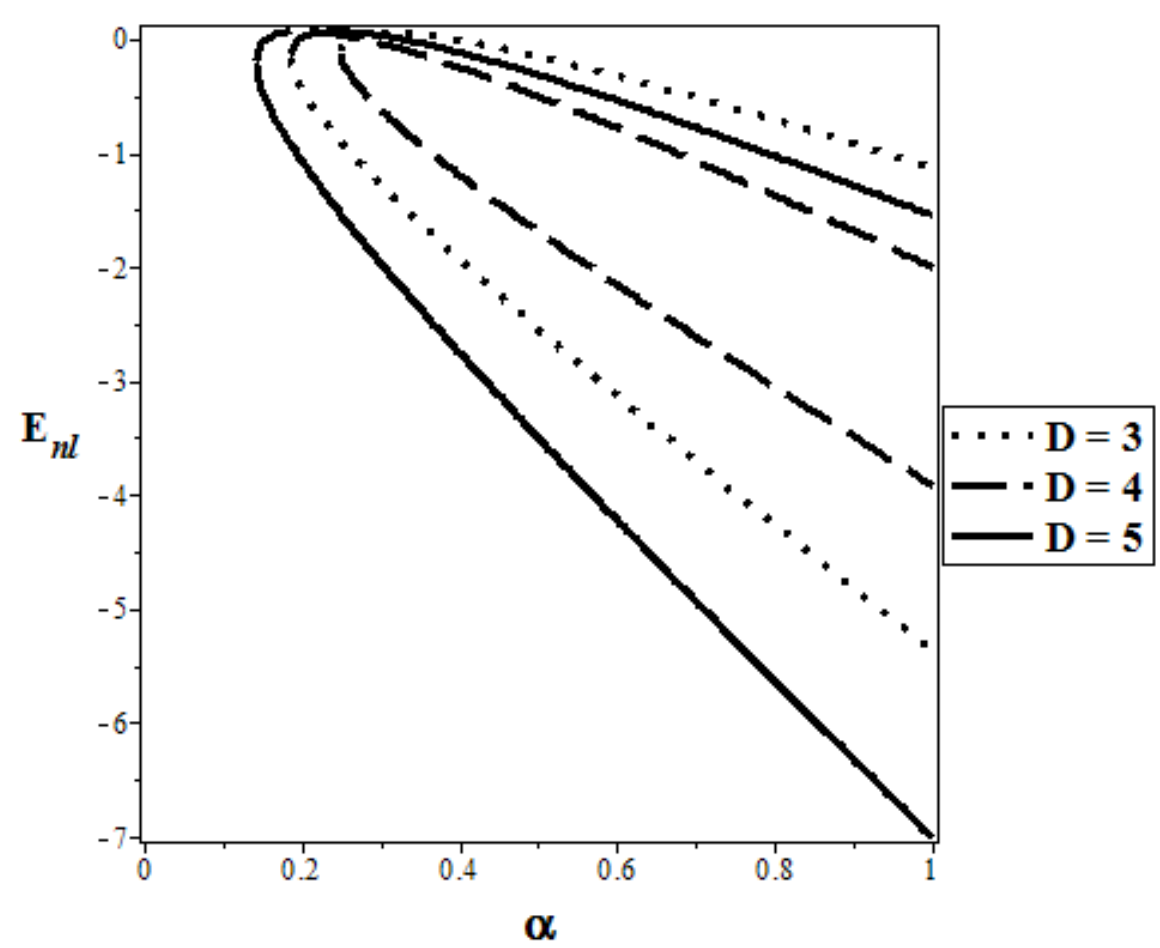

Figure 3. Energy Eigenvalue variation with $\alpha$ for different dimensions with $g=-1, A=5, n=2, \ell=1$. 


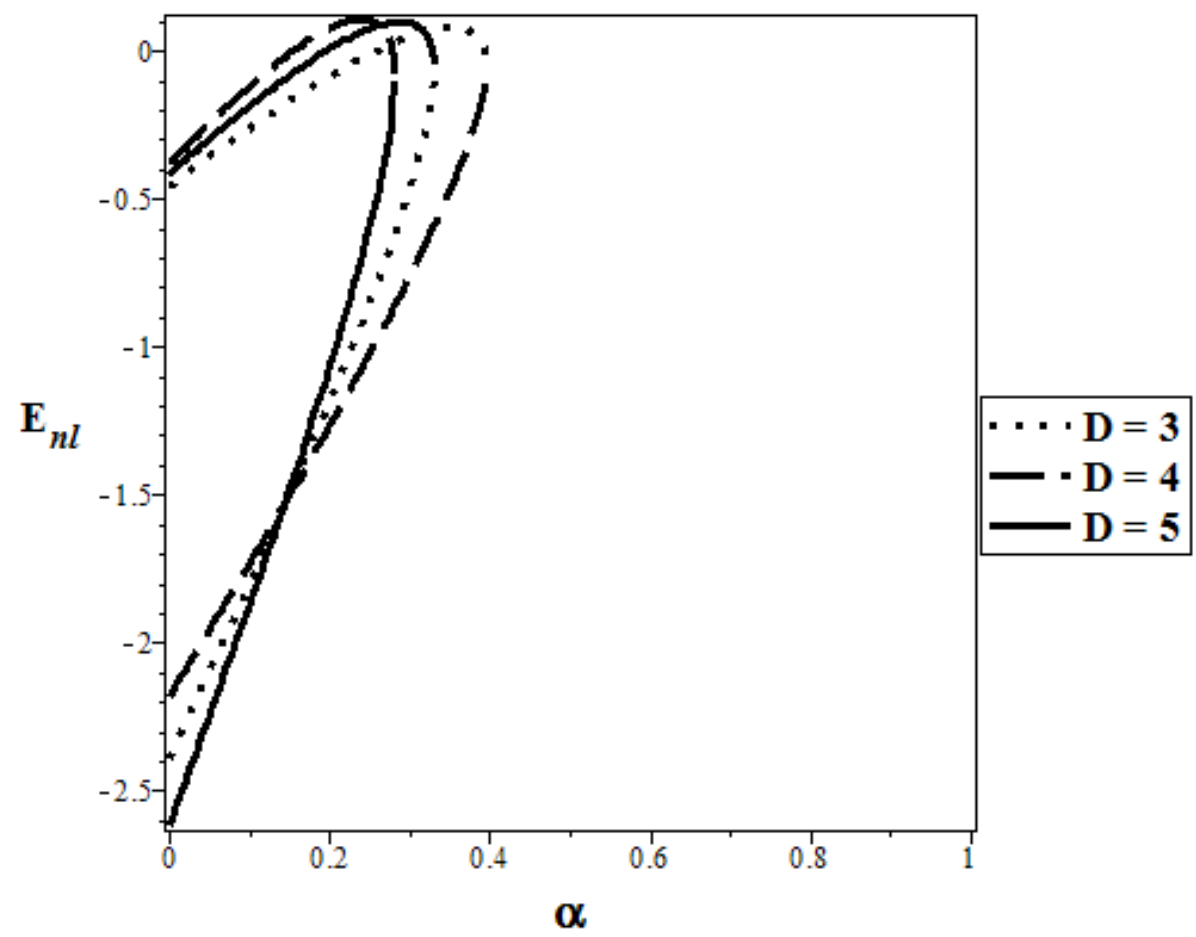

Figure 4. Energy Eigenvalue Variation with $\alpha$ for different dimensions with $g=1, A=5, n=2, \ell=1$.

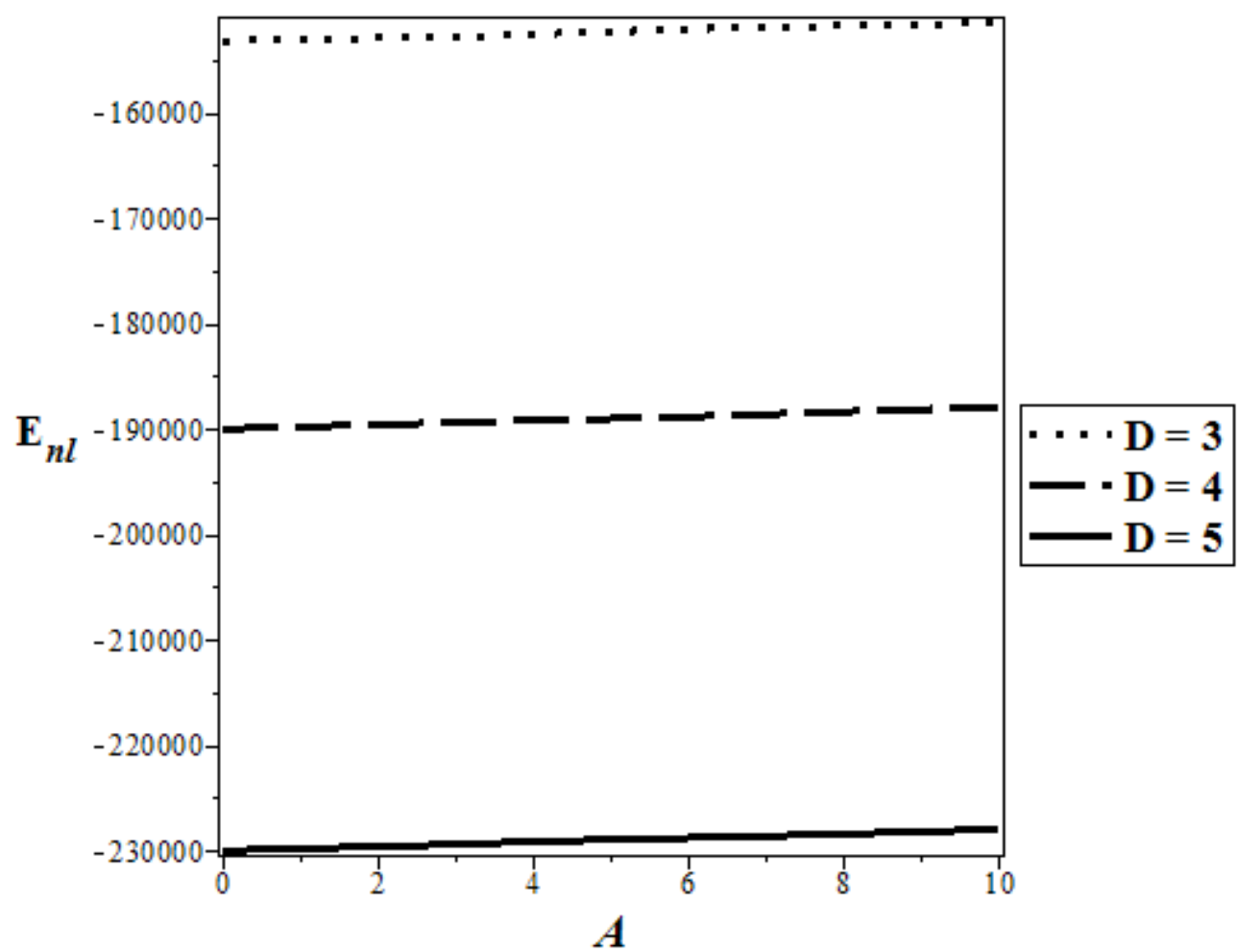

Figure 5. Energy Eigenvalue Variation with $A$ for different dimensions with $g=0, \alpha=100, n=2, \ell=1$. 


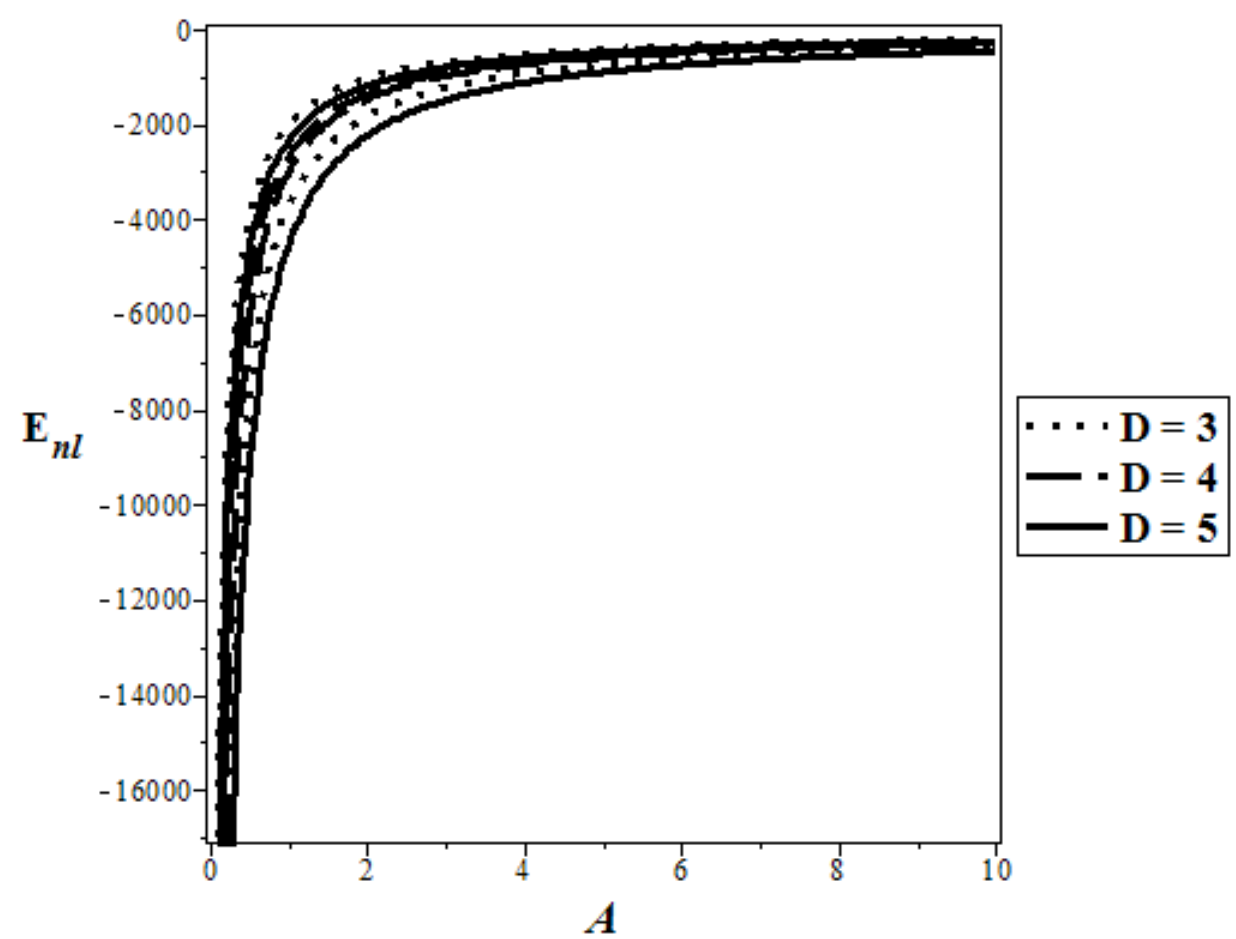

Figure 6. Energy Eigenvalue Variation with $A$ for different dimensions with $g=-1, \alpha=100, n=2, \ell=1$.

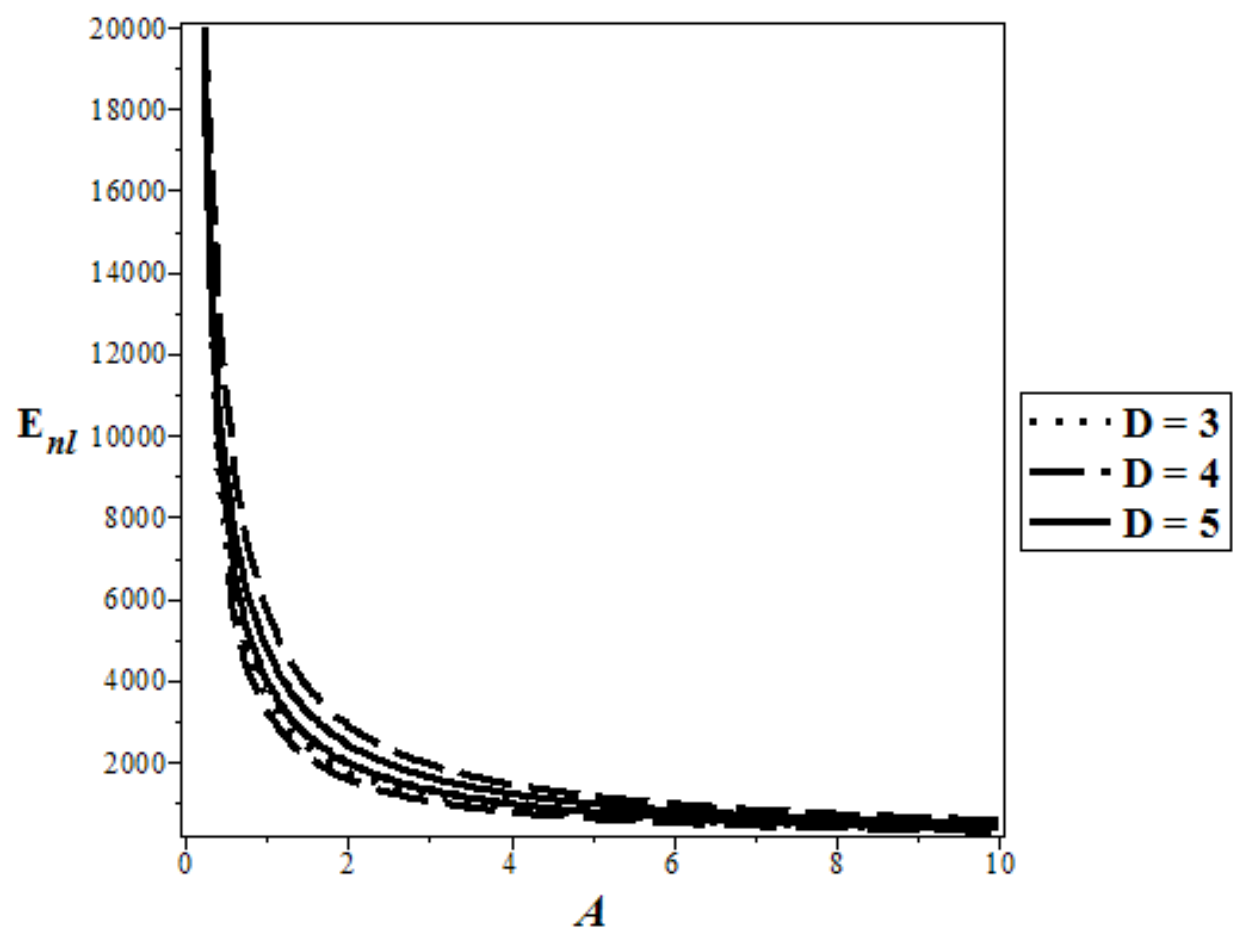

Figure 7. Energy Eigenvalue Variation with $A$ for different dimensions with $g=1, \alpha=100, n=2, \ell=1$. 


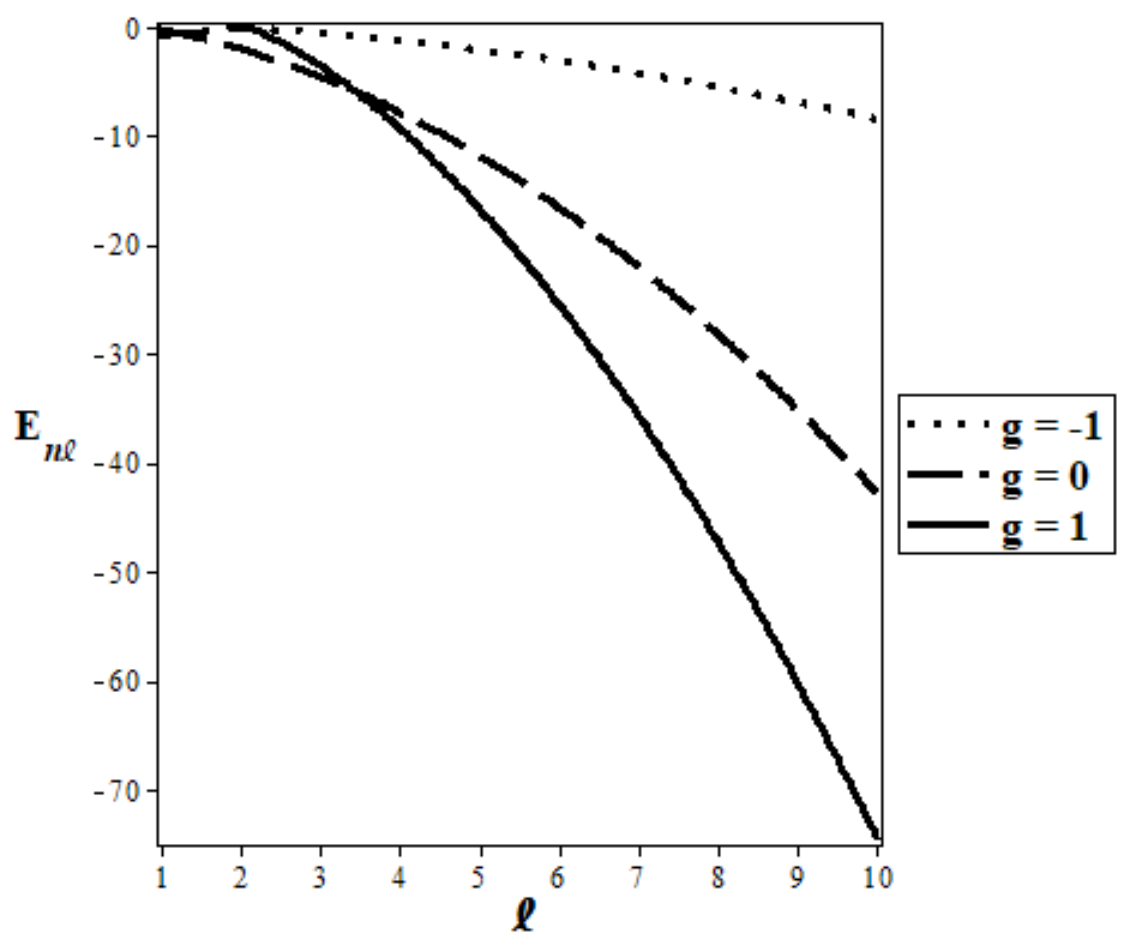

Figure 8. Energy Eigenvalue Variation with $\ell$ for different values of $g$ with $D=3, \alpha=1, A=5, n=0$.

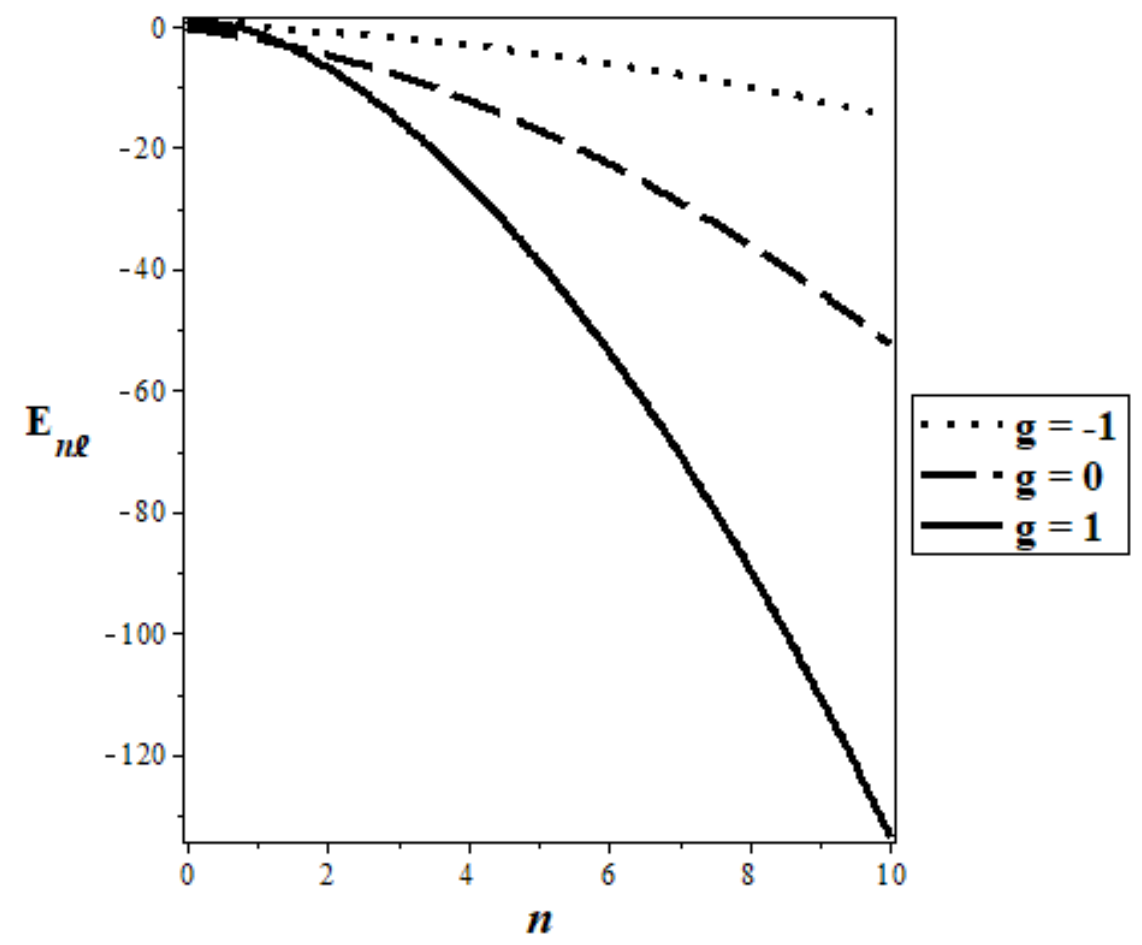

Figure 9. Energy Eigenvalue Variation with $n$ for different values of $g$ with

$$
D=3, \alpha=1, A=5, \ell=1 \text {. }
$$




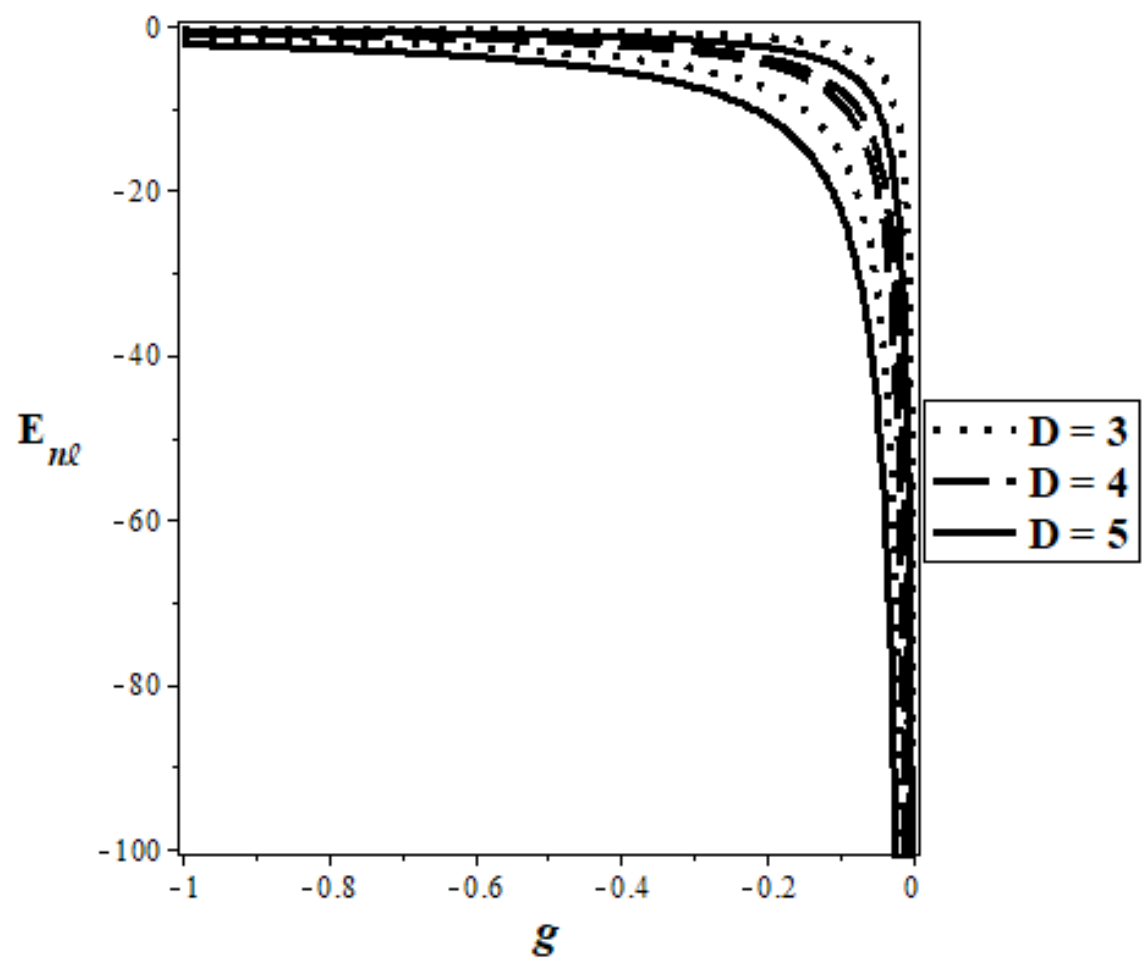

Figure 10. Energy Eigenvalue Variation with $g$ for different dimensions, with $\alpha=10, A=100, n=2, \ell=1$

\section{Conclusions}

We have solved the Schrödinger equation with the energy-dependent screened Coulomb potential in higher dimensions. With the use of the conventional Nikiforov-Uvarov method and a new form of Greene-Aldrich approximation, the energy eigenvalues and its corresponding eigenfunctions were obtained approximately. Numerical values of the energy eigenvalues were also obtained with natural units, in three dimensions. We have also elucidated the variation of these energy spectra with different potential parameters and the energy slope parameter. The effect of the energy slope parameter is clearly seen in the existence of duo energy spectra, as compared to when the energy slope parameter is diminished. Special cases have been deduced, and these results agree perfectly with available literature.

\section{Acknowledgments}

The authors thank the kind reviewers for the positive comments and suggestions that lead to an improvement of our manuscript

\section{References}

[1] Chun-Feng, H., Zhong-Xiang, Z., Yan, L., Bound states of the Klein-Gordon equation with vector and scalar Wood-Saxon potentials, Acta Physica Sinica (Overseas Edition) $8 \quad$ (8) (1999) 561. https://doi.org/10.1088/1004-423X/8/8/001.

[2] Sever, R., Tezan, C., Yeșiltaş, Ö., Bucurgat, M., Exact Solution of Effective Mass Schrödinger Equation for the Hulthen Potential, International Journal of Theoretical Physics 49 (9) (2008) 2243-2248. https://doi.org/10.1007/s10773-008-9656-7.

[3] Yahya, W. A., Oyewumi, K. J., Thermodynamic properties and approximate solutions of the $\ell$-state Pöschl-Teller-type potential, Journal of the Association of Arab Univ. for Basic and Applied $\begin{array}{lllll}\text { Sciences } & 21 & \text { (1) } & \text { (2016) }\end{array}$ https://doi.org/10.1016/j.jaubas.2015.04.001.

[4] Sun, Y., He, S., Jia, C.-S., Equivalence of the deformed modified Rosen-Morse potential energy 
model and the Tietz potential energy model, Physica $\begin{array}{lllll}\text { Scripta } & 87 & \text { (2) } & \text { (2013) } & 025301 .\end{array}$ https://doi.org/10.1088/0031-8949/87/02/025301.

[5] Onate, C. A., Idiodi, J. O. A., Eigensolutions of the Schrödinger Equation with Some Physical Potentials, Chinese Journal of Physics 53 (7) (2015) 120001-1120001-10. https://doi.org/10.6122/CJP.20150831E.

[6] Antia, A. D., Ikot, A. N., Hassanabadi, H., Maghsoodi, E., Bound state solutions of Klein-Gordon equation with Mobius square plus Yukawa potentials, Indian Journal of Physics 87 (11) (2013) 1133-1139. https://doi.org/10.1007/s12648-013-0336-y.

[7] Ikot, A. N., Awoga, O. A., Hassanabadi, H., Maghsoodi, E., Analytical Approximate Solution of Schrödinger Equation in $D$ Dimensions with Quadratic Exponential-Type Potential for Arbitrary l-State, Communications in Theoretical Physics 61 (4) (2014) 457-463. https://doi.org/10.1088/0253-6102/61/4/09.

[8] Falaye, B. J., Oyewumi, K. J., Abbas, M., Exact solution of Schrödinger equation with q-deformed quantum potentials using Nikiforov-Uvarov method, Chinese Physics B 22 (11) (2013) 110301. https://doi.org/10.1088/1674-1056/22/11/110301.

[9] Ciftci, H., Hall, R. L., Saad, N., Asymptotic iteration method for eigenvalue problems, Journal of Physics A: Mathematical and General 36 (47) (2003) 11807-11816. https://doi.org/10.1088/03054470/36/47/008.

[10] Falaye, B. J., Any $l$-state solutions of the Eckart potential via asymptotic iteration method, Central European Journal Physics 10 (4) (2012) 960965. https://doi.org/10.2478/s11534-012-0047-6.

[11] Setare, M. R., Karimi, E., Algebraic approach to the Kratzer potential, Physica Scripta 75 (1) (2007) 9093. https://doi.org/10.1088/0031-8949/75/1/015.

[12] Qiang, W. C., Dong, S. H., Proper quantization rule, Europhysics Letters 89 (1) (2010) 10003. https://doi.org/10.1209/0295-5075/89/10003.

[13] Ikhdair, S. M., Sever, R., Exact quantization rule to the Kratzer-type potentials: an application to the diatomic molecules, Journal of Mathematical $\begin{array}{lllll}\text { Chemistry } & 45 & \text { (4) } & \text { (2009) } & 1137 .\end{array}$ https://doi.org/10.1007/s10910-008-9438-8.

[14] Chen, G., The exact solutions of the Schrödinger equation with the Morse potential via Laplace transforms, Physics Letters A 326 (1-2) (2004) 55-57. https://doi.org/10.1016/j.physleta.2004.04.029.
[15] Onate, C. A., Ojonubah, J. O., Eigensolutions of the Schrödinger equation with a class of Yukawa potentials via supersymmetric approach, Journal of Theoretical and Applied Physics 10 (1) (2016) 21-26. https://doi.org/10.1007/s40094-015-0196-2.

[16] Ikot, A. N., Obong, H. P., Abbey, T. M., Zare, S., Ghafourian, M., Hassanabadi, H., Bound and Scattering State of Position Dependent Mass KleinGordon Equation with Hulthen Plus Deformed-Type Hyperbolic Potential, Few-Body Systems 57 (9) (2016) 807-822. https://doi.org/10.1007/s00601-016-1111-3.

[17] Onate, C. A., Onyeaju, M. C., Ikot, A. N., Ojonubah, J. O., Analytical solutions of the KleinGordon equation with a Combined potential, Chinese Journal of Physics 54 (5) (2016) 820-829. https://doi.org/10.1016/j.cjph.2016.08.007.

[18] Onate, C. A., Ikot, A. N., Onyeaju, M. C., Udoh M. E., Bound state solutions of D-dimensional KleinGordon equation with hyperbolic potential, Karbala International Journal of Modern Science 3 (1) (2017) 1-7. https://doi.org/10.1016/j.kijoms.2016.12.001.

[19] Okorie, U. S., Ibekwe, E. E., Onyeaju, M. C., Ikot, A. N., Solutions of the Dirac and Schrödinger equations with shifted Tietz-Wei potential, The European Physical Journal Plus 133 (10) (2018) 433. https://doi.org/10.1140/epjp/i2018-12307-4.

[20] Okorie, U. S., Ikot, A. N., Edet, C. O., Akpan, I. O., Sever, R., Rampho, G. J., Solutions of the Klein Gordon equation with generalized hyperbolic potential in D-dimensions, Journal of Physics Communications 3 (9) (2019) 095015. https://doi.org/10.1088/23996528/ab42c6.

[21] Dong, S.-H., Factorization Method in Quantum Mechanics, Springer, Dordrecht, 2007. https://doi.org/10.1007/978-1-4020-5796-0.

[22] Jia, C.-S., Jia, Y., Relativistic rotation-vibrational energies for the $\mathrm{Cs}_{2}$ molecule, The European Physical Journal D $71 \quad$ (1) (2017) 3. https://doi.org/10.1140/epjd/e2016-70415-y.

[23] Dong, S., Sun, G.-H., Dong, S.-H., Arbitrary $l$ Wave Solutions of the Schrödinger Equation for the Screen Coulomb Potential, International Journal of Modern Physics E 22 (6) (2013) 1350036. https://doi.org/10.1142/S0218301313500365.

[24] Ikhdair, S. M., Sever, R., A perturbative treatment for the bound states of the Hellmann potential, Journal of Molecular Structure: THEOCHEM 809 (1-3) (2007) 103-113.

https://doi.org/10.1016/j.theochem.2007.01.019. 
[25] Liverts, E. Z., Drukarev, E. G., Krivec, R., Mandelzweig, V. B., Analytic presentation of a solution of the Schrödinger equation, Few-Body Systems $44 \quad(1-4) \quad$ (2008) 367-370. https://doi.org/10.1007/s00601-008-0328-1.

[26] Maghsoodi, E., Hassanabadi, H., Aydoğdu, O., Dirac particles in the presence of the Yukawa potential plus a tensor interaction in SUSYQM framework, Physica Scripta $86 \quad$ (1) (2012) 015005. https://doi.org/10.1088/0031-8949/86/01/015005.

[27] Edwards, J. P., Gerber, U., Schubert, C., Trejo, M. A., Weber, A., The Yukawa potential: ground state energy and critical screening, Progress of Theoretical and Experimental Physics 2017 (8) (2017) 083A01. https://doi.org/10.1093/ptep/ptx107.

[28] Hamzavi, H., Movahedi, M., Thylwe, K.-E., Rajabi, A. A., Approximate Analytical Solution of the Yukawa Potential with Arbitrary Angular Momenta, Chinese Physics Letters 29 (8) (2012) 080302. https://doi.org/10.1088/0256-307X/29/8/080302.

[29] Garcia-Martínez, J., García-Ravelo, J., Peña, J. J., Schulze-Halberg, A., Exactly solvable energydependent potentials, Physics Letters A 373 (40) (2009) https://doi.org/10.1016/j.physleta.2009.08.012.

[30] Yekken, R., Lombard, R. J., Energy-dependent potentials and the problem of the equivalent local potential, Journal of Physics A: Mathematical and $\begin{array}{llll}\text { Theoretical } & 43 & \text { (2010) } & 125301 .\end{array}$ https://doi.org/10.1088/1751-8113/43/12/125301.

[31] Yekken, R., Lassaut, M., Lambard, R. J., Applying supersymmetry to energy dependent potentials, Annals of Physics 338 (2013) 195-206. https://doi.org/10.1016/j.aop.2013.08.005.

[32] Hassanabadi, H., Zarrinkamar, S., Rajabi, A. A., Exact Solutions of $D$-Dimensional Schrödinger Equation for an Energy-Dependent Potential by NU Method, Communications in Theoretical Physics 55 (2011) 541-544. https://doi.org/10.1088/0253$6102 / 55 / 4 / 01$

[33] Hassanabadi, H., Zarrinkamar, S., Hamzavi, H., Rajabi, A. A., Exact Solutions of D-Dimensional Klein-Gordon Equation with an Energy-Dependent Potential by Using of Nikiforov-Uvarov Method, Arabian Journal for Science and Engineering 37 (2012) 209-215. https://doi.org/10.1007/s13369-011-0168-z.
[34] Lombard, R. J., Mareš, J., Volpe, C., Wave equation with energy-dependent potentials for confined systems, Journal of Physics G: Nuclear and Particle

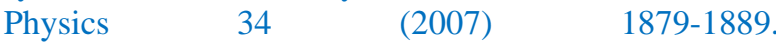
https://doi.org/10.1088/0954-3899/34/9/002.

[35] Lombard, R. J., Mares, J., The many-body problem with an energy-dependent confining potential, Physics Letters A 373 (4) (2009) 426-429. https://doi.org/10.1016/j.physleta.2008.12.009.

[36] Gupta, P., Mehrotra, I., Study of Heavy Quarkonium with Energy Dependent Potential, Journal of Modern Physics 3 (10) (2012) 1530-1536. https://doi.org/10.4236/jmp.2012.310189.

[37] Budaca, R., Bohr Hamiltonian with an energydependent $\gamma$-unstable Coulomb-like potential, The European Physical Journal A 52 (2016) 314. https://doi.org/10.1140/epja/i2016-16314-8.

[38] Boumali, A., Labidi, M., Shannon entropy and Fisher information of the one-dimensional KleinGordon oscillator with energy-dependent potential, Modern Physics Letters A 33 (6) (2018) 1850033. https://doi.org/10.1142/S0217732318500335.

[39] Hassanabadi, H., Yazarloo, B. H., Zarrinkamar, S., Rahimov, H., Deng-Fan Potential for Relativistic Spinless Particles — an Ansatz Solution, Communications in Theoretical Physics 57 (3) (2012) 339-342. https://doi.org/10.1088/0253-6102/57/3/02.

[40] Nikiforov, A. F., Uvarov, V. B., Special Functions of Mathematical Physics, Basel, Birkhäuser, 1998.

[41] Birkdemir, C., Application of the NikiforovUvarov Method in Quantum Mechanics, In: Theoretical Concept of Quantum Mechanics, Pahlavani, M. R., ed., InTech: Rijeka, Croatia, 2012, Ch. 11. https://doi.org/10.5772/33510.

[42] Abramowitz, M., Stegun, I. A., Handbook of Mathematical Functions with Formulas, Graphs and Mathematical Tables, U.S. Government Printing Office, Washington, 1964. http://people.math.sfu.ca/ cbm/aands/abramowitz_and _stegun.pdf. 


\section{Appendix: Review of Nikiforov-Uvarov (NU) method}

According to Nikiforov and Uvarov ${ }^{40}$, the NU method transforms Schrödinger-like equations into a second order differential equation using a coordinate transformation $z=z(r)$, which is given by Eq. A1.

$\psi^{, \frac{\tilde{\tau}(z)}{\sigma(z)}} \frac{\hat{\sigma}(z)}{\sigma^{2}(z)}$

Here, $\tilde{\sigma}(z), \sigma(z)$ are polynomials of at most second degree, and $\tilde{\tau}(z)$ is a first-degree polynomial. By employing the transformation (Eq. A2),

$\psi(z)=\Phi(z) y_{n}(z)$

we obtain the exact solution of Eq. A1 in a form of hypergeometric-type equation given by Eq. A3.

$\sigma(z) y_{n}^{\prime \prime}(z)+\tau(z) y_{n}^{\prime}(z)+\lambda y_{n}(z)=0$

Let us define the logarithm derivative function $\Phi(z)$ as ${ }^{40}$ (Eq. A4).

$\frac{\Phi^{\prime}(z)}{\Phi(z)}=\frac{\pi(z)}{\sigma(z)}$

where $\pi(z)$ is at most a first-degree polynomial. The second part of $\psi(z)$ being $y_{n}(z)$ in Eq. A2, is the hypergeometric function with its polynomial solution given by Rodrigues relation (Eq. A5)

$y_{n}(z)=\frac{B_{n}}{\rho(z)} \frac{d^{n}}{d z^{n}}\left[\sigma^{n}(z) \rho(z)\right]$

Let us mention here that $B_{n}$ is the normalization constant and $\rho(z)$ is the weight function which must satisfy the condition expressed by Eq. A6.

$\frac{d}{d z}[\sigma(z) \rho(z)]=\tau(z) \rho(z)$

with Eq. A7

$\tau(z)=\tilde{\tau}(z)+2 \pi(z)$

The eigenfunctions and eigenvalues can be obtained using the definition of the following function $\pi(z)$ and parameter $\lambda$ as shown (Eq. A8):

$\pi(z)=\frac{\sigma^{\prime}(z)-\tilde{\tau}(z)}{2} \pm \sqrt{\left(\frac{\sigma^{\prime}(z)-\tilde{\tau}(z)}{2}\right)^{2}-\tilde{\sigma}(z)+k \sigma(z)}$

and (Eq. A9)

$\lambda=k+\pi^{\prime}(z)$

To obtain the value of $k$, we set the discriminant of the square root in Eq. A8 equal to zero. As such, the new eigenvalue equation (Eq. A10) is obtained as

$\lambda+n \tau^{\prime}(z)+\frac{n(n-1)}{2} \sigma^{\prime \prime}(z)=0,(n=0,1,2, \ldots)$ 